\title{
This Month in APR
}

\section{By Yu-Kyoung Oh, Associate Editor}

\section{Hepatocyte-targeting and sustained gene deliv- ery}

The article focused in this issue (Jiang et al., 2010) attracted my attention due to targeted and sustained gene delivery using sugar-modified cationic polymers and in situ-gelling hydrogels. For efficient gene therapy, it would be desirable to achieve delivery to the target cells and extended expression of the therapeutic proteins over prolonged periods. The highly polar and macromolecular nature of plasmid DNA limit cellular uptake in vitro, and the rapid degradation of nucleic acids by nucleases shorten the half-life in vivo. The negligible cellular uptake and instability of systemically administered nucleic acids requires the use of viral or nonviral gene delivery systems for in vivo applications.

As cationic nonviral gene delivery systems, cationic polymers and lipid-based nanoparticles have been studied to enhance the cellular delivery (Al-Dosari and Gao, 2009). Among cationic polymers, polyethylenimine (PEI) and chitosan have been widely studied as gene delivery systems. PEI has been reported to enhance the endosomal escaping and nuclear import of plasmid DNA (Zhou et al., 2007), and prolong the mean residence time of plasmid DNA in vivo (Jeong et al., 2007). Although the cationic gene delivery systems such as PEI have several advantages over viral vectors in terms of simplicity, less immunogenicity, and lower potential of mutagenesis, PEI suffers from the insufficient transfection efficiency, the lack of biodegradability and targeting capability. The hybrid system of chitosan and PEI has been shown to improve the transfection efficiency of PEI (Jiang et al., 2008a). Although substantial progresses have been made to improve the transfection efficiencies, cationic delivery systems of future generation are still required to possess targeting ligands for organ-specific distribution and improve target cell-directed uptake of plasmid DNA.

Given the clinical significance of liver-related diseases, the liver or hepatocyte-targeting strategies have been widely studied. Sugars have been used to direct the delivery of genes to livers or hepatocytes. Several groups reported the hepatocyte-targeting properties of galactose-modified cationic gene delivery systems (Sagara and Kim, 2002; Jiang et al., 2008b). These targeting moieties not only provide the specificity of gene delivery to the cells with higher expression of unique receptors, but also increase the efficiency of cellular uptake via receptor-mediated endocytosis (Kim et al., 2007). The featured article (Jiang et al., 2010) consistently showed the enhanced cellular activity of luciferase after delivery using galactosylated chitosan-g-PEI as compared to PEI alone.

Besides targeted delivery, the duration of target gene expression remains as another hurdle in this field.

Hydrogels have been used to deliver macromolecules over sustained periods. Thermosensitive hydrogels may be useful for the formulation of injectable hydrogels due to the conversion of sol to gel in the body temperature. The temperature sensitive properties of

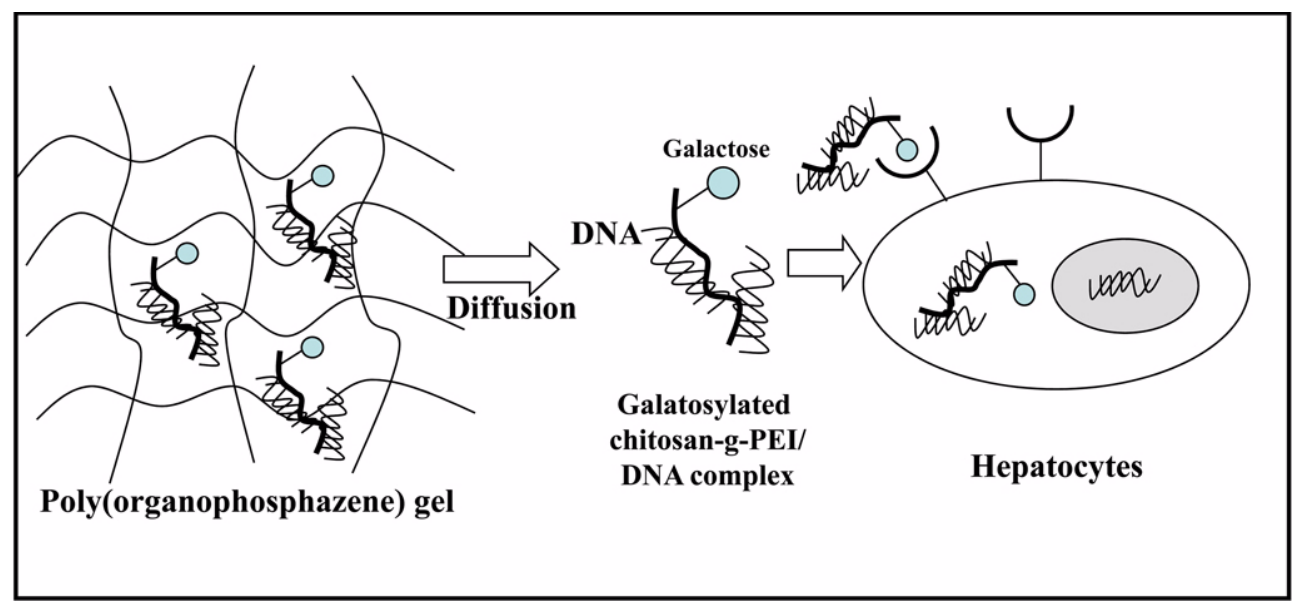

Fig. 1. Sustained and hepatocyte-targeted delivery of plasmid DNA using galactosylated chitosan-g-PEI polymers dispersed in thermosensitive poly(organophosphazene) hydrogel matrix. 
hydrogels have been utilized for controlling the release of plasmid DNA by external control of temperature changes. Poly(organophosphazene) hydrogels have been used for local delivery of anticancer drugs (Kang et al., 2006), proteins (Kang and Song, 2008), and mesenchymal stem cells (Chun et al., 2009). Despite the advantages of in situ-gelling, biodegradable, and less toxic properties, poly(organophosphazene) hydrogels have been little used for delivery of plasmid DNA. The current article of Jiang et al. (2010) showed the new application of poly(organophosphazene) hydrogel for sustained in vivo delivery of galactosylated chitosan-gPEI.

Here, the authors used intraperitoneal route for prolonged retention of poly(organophosphazene) hydrogels in vivo. They showed the time-dependent release of plasmid DNA from the hydrogel matrix over two weeks. The authors provided different biodistribution patterns between PEI/DNA and galactosylated chitosan-g-PEI/ DNA complexes. From the data described in the articles, it is not clear whether the in vivo liver-targeting was indeed achieved in hepatocyte levels. Moreover, they need to demonstrate the efficiency of target gene expressions in the liver tissues, the duration of gene expression, and in vivo toxicity of poly(organophosphazene)hydrogels in the future.

Although further studies are needed to clarify the value of poly(organophosphazene)hydrogels for prolonged hepatocyte gene delivery in vivo, the featured article is meaningful in that it opened a new way to apply the themosensitive hydrogels for sustained and targeted gene delivery.

\section{REFERENCES}

Al-Dosari, M. S. and Gao, X., Nonviral gene delivery: principle, limitations, and recent progress, AAPS J., 11, 671681 (2009).

Chun, C., Lim, H. J., Hong, K. Y., Park, K. H., and Song, S. C., The use of injectable, thermosensitive poly(organophosphazene)-RGD conjugates for the enhancement of mesenchymal stem cell osteogenic differentiation. Bio- materials, 30, 6295-6308 (2009).

Jeong, G. J., Byun, H. M., Kim, J. M., Yoon, H., Choi, H. G., Kim, W. K., Kim, S. J., and Oh, Y. K., Biodistribution and tissue expression kinetics of plasmid DNA complexed with polyethylenimines of different molecular weight and structure. J. Control. Release, 118, 118-125 (2007).

Jiang, H. L., Kim, T. H., Kim, Y. K., Park, I. Y., Cho, M. H., and Cho, C. S., Efficient gene delivery using chitosanpolyethylenimine hybrid systems. Biomed. Mater., 3, 25013 (2008a).

Jiang, H. L., Kwon, J. T., Kim, E. M., Kim, Y. K., Arote, R., Jere, D., Jeong, H. J., Jang, M. K., Nah, J. W., Xu, C. X., Park, I. K., Cho, M. H., and Cho, C. S., Galactosylated poly (ethylene glycol)-chitosan-graft-polyethylenimine as a gene carrier for hepatocyte-targeting. J. Control. Release, 131,150-157 (2008b).

Jiang, H. L., Kim, Y.-K., Lee, S.-M., Park, M.-R., Kim, E.-M., Jin, Y.-M., Arote, R., Jeong, H.-J., Song, S.-C., Cho, M.-H., and Cho, C.-S., Galactosylated chitosan-g-PEI/DNA complexes-loaded poly(organophosphazene) hydrogel as a hepatocyte targeting gene delivery system in vitro and in vivo. Arch. Pharm. Res., 33, 551-556 (2010).

Kang, G. D., Cheon, S. H., and Song, S. C., Controlled release of doxorubicin from thermosensitive poly(organophosphazene) hydrogels. Int. J. Pharm., 319, 29-36 (2006).

Kang, G. D. and Song, S. C., Effect of chitosan on the release of protein from thermosensitive poly(organophosphazene) hydrogels. Int. J. Pharm., 349, 188-195 (2008).

Kim, T. H., Jiang, H. L., Nah, J. W., Cho, M. H., Akaike, T., and Cho, C. S., Receptor-mediated gene delivery using chemically modified chitosan. Biomed. Mater., 2, S95-100 (2007).

Sagara, K. and Kim, S. W., A new synthesis of galactose-poly (ethylene glycol)-polyethylenimine for gene delivery to hepatocytes. J. Control. Release, 79, 271-281 (2002).

Zhou, J., Yockman, J. W., Kim, S. W., and Kern, S. E., Intracellular kinetics of non-viral gene delivery using polyethylenimine carriers. Pharm. Res., 24, 1079-1087 (2007).

College of Pharmacy, Seoul National University, Seoul 151742, South Korea (ohyk@snu.ac.kr)

See page 551-556. 